J. Kaivers' ', C. Rautenberg', B. Betz², P. Czyborra ${ }^{3}$, R. Haas' ${ }^{1}$, M. Rudelius ${ }^{4}$, M. Lübbert ${ }^{5}$, G. Kobbe', U. Germing'

${ }^{1}$ Klinik für Hämatologie, Onkologie und klinische Immunologie, Universitätsklinikum Düsseldorf, Düsseldorf,

Deutschland

${ }^{2}$ Institut für Humangenetik, Universitätsklinikum Düsseldorf, Düsseldorf, Deutschland

${ }^{3}$ MVZ Onkologie Essen, Essen, Deutschland

${ }^{4}$ Medizinische Fakultät, Pathologisches Institut, Ludwig-Maximilians-Universität München, München,

Deutschland

${ }^{5}$ Klinik für Hämatologie, Onkologie und Stammzelltransplantation, Universitätsklinik Freiburg, Freiburg im

Breisgau, Deutschland

\title{
Erratum zu: Diagnostik und Therapie der chronischen myelomonozytären Leukämie im Jahr 2020
}

\section{Erratum zu:}

best practice onkologie 2020

https://doi.org/10.1007/s11654-020-00278-5

Im Originalbeitrag ist Tab. 5 leider fehlerhaft. Nachfolgend die

Tabelle mit korrigierter Legende.

\begin{tabular}{|c|c|c|c|c|c|}
\hline \multicolumn{6}{|c|}{ Berechnung des genetischen Scores } \\
\hline- & Zytogenetische Risikogruppe nach CPSS & ASXL1 & NRAS & RUNX1 & SETBP1 \\
\hline \multicolumn{6}{|c|}{ Score } \\
\hline 0 & Niedrig & Wildtyp & Wildtyp & Wildtyp & Wildtyp \\
\hline 1 & Intermediär & Mutiert & Mutiert & - & Mutiert \\
\hline 2 & Hoch & - & - & Mutiert & - \\
\hline \multicolumn{6}{|c|}{ Berechnung des gesamten Scores } \\
\hline \multicolumn{2}{|c|}{-} & $\begin{array}{l}\text { Genetische Risiko- } \\
\text { gruppe }\end{array}$ & $\begin{array}{l}\text { Blastengehalt im } \\
\text { Knochenmark (\%) }\end{array}$ & Leukozyten & $\begin{array}{l}\text { Transfusionsbedarf für } \\
\text { Erythrozytenkonzentrate }\end{array}$ \\
\hline \multicolumn{6}{|c|}{ Score } \\
\hline \multicolumn{2}{|l|}{0} & Niedrig & $<5 \%$ & $<13.000 / \mu l$ & Nein \\
\hline \multicolumn{2}{|c|}{1} & Intermediär-I & $\geq 5 \%$ & $\geq 13.000 / \mu l$ & $\mathrm{Ja}$ \\
\hline \multicolumn{2}{|c|}{2} & Intermediär-II & - & - & - \\
\hline \multicolumn{2}{|c|}{3} & Hoch & - & - & - \\
\hline \multicolumn{6}{|c|}{$\begin{array}{l}\text { aCMML-spezifische zytogenetische Risikoeinteilung gemäß Such et al. [1]: niedrig: normaler Karyotyp; isolierter Verlust des Y-Chromosoms; intermediär: andere Aberratio- } \\
\text { nen; hoch: Trisomie 8, komplexer Karyotyp ( } \geq 3 \text { Aberrationen), Aberrationen auf Chromosom 7; Genetische Risikogruppen: niedrig (0), intermediär-I (1), intermediär-II (2), } \\
\text { hoch ( } \geq 3 \text { ) } \\
\text { bTransfusion von Erythrozytenkonzentraten: mindestens } 1 \text { Transfusion alle } 8 \text { Wochen für } 4 \text { Monate }\end{array}$} \\
\hline
\end{tabular}

Die Online-Version des Originalartikels ist unter https://doi.org/10.1007/

s11654-020-00278-5 zu finden.

best practice onkologie 2021 - 16 (3): 126-128

https://doi.org/10.1007/s11654-021-00287-y

Online publiziert: 2. Februar 2021

(c) Springer Medizin Verlag GmbH, ein Teil von Springer Nature 2021

126 best practice onkologie $3 \cdot 2021$ 
Literatur

1. Such E, Germing U, Malcovati L, Cervera J, Kuendgen A, Della Porta MG, Nomdedeu B, Arenillas L, Luño E, Xicoy B, Amigo ML, Valcarcel D, Nachtkamp K, Ambaglio I, Hildebrandt B, Lorenzo I, Cazzola M, Sanz G (2013) Development and validation of a prognostic scoring system for patients with chronic myelomonocytic leukemia. Blood 121(15):3005-3015

2. Elena C, Galli A, Such E, Meggendorfer M, Germing U, Rizzo E, Cervera J, Molteni E, Fasan A, Schuler E, Ambaglio I, Lopez-Pavia M, Zibellini S, Kuendgen A, Travaglino E, Sancho-Tello R, Catricalà S, Vicente Al, Haferlach T, Haferlach C, Sanz GF, Malcovati L, Cazzola M (2016) Integrating clinical features and genetic lesions in the risk assessment of patients with chronic myelomonocytic leukemia. Blood 128(10):1408-1417

\section{Korrespondenzadresse}

\section{Dr. J. Kaivers}

Klinik für Hämatologie, Onkologie und klinische Immunologie,

Universitätsklinikum Düsseldorf

Moorenstr. 5, 40225 Düsseldorf, Deutschland

jennifer.kaivers@med.uni-duesseldorf.de

\section{CME-Punkte sammeln in 3 Schritten}

Als Zeitschriftenabonnent stehen Ihnen unter CME.SpringerMedizin.de alle zertifizierten Fortbildungskurse Ihrer Zeitschrift zur Verfügung.

So einfach sammeln Sie CME-Punkte:

\section{Registrieren}

Um CME-Fortbildungen bearbeiten zu können, müssen Sie sich einmalig unter www.springermedizin.de/register registrieren. Bitte geben Sie bei der Registrierung die Lieferadresse Ihrer abonnierten Zeitschrift an, damit die Angaben Ihres OnlineAccounts mit den Angaben Ihres Zeitschriften-Abonnements übereinstimmen. Die CME-Beiträge werden anschließend automatisch freigeschaltet.

\section{Beitrag auswählen}

Sobald Sie sich mit Ihren Zugangsdaten angemeldet haben, können Sie auf CME.SpringerMedizin.de die gewünschten CME-Kurse Ihrer Zeitschrift nutzen. Die Kurse können jederzeit unterbrochen und später fortgesetzt werden.

\section{CME-Punkte sammeln}

Zu jedem Fortbildungskurs gehört ein Fragebogen mit 10 CMEFragen. Mit 7 richtigen Antworten haben Sie bestanden und erhalten umgehend eine Teilnahmebescheinigung!

Teilnehmen und weitere Informationen unter: CME.SpringerMedizin.de

Unser Tipp: Noch mehr Fortbildung bieten die e.Med-KombiAbos. Hier stehen Ihnen die CME-Kurse der Fachzeitschriften von Springer Medizin in elektronischer Form zur Verfügung. Auf Wunsch erhalten sie mit den e.Med-Kombi-Abos außerdem eine gedruckte Fachzeitschrift Ihrer Wahl.

Testen Sie e.Med kostenlos und unverbindlich!

Jetzt informieren unter

www.springermedizin.de $\Rightarrow$ "Abo-Shop" oder telefonisch unter 0800-77 80777

(Montag bis Freitag, 10 bis $17 \mathrm{Uhr}$ ) 
Hier steht eine Anzeige.

\section{Springer}

